\title{
Stability and Trapping of Slow Hydromagnetic Waves in Rotating Cylindrical Geometry
}

\author{
Steven D. LONDON* \\ Department of Applied Mathematical Sciences, University of Houston-Downtown, \\ One Main Street Houston, TX 77002, U.S.A.
}

(Received November 24, 1992; Revised April 1, 1993; Accepted April 24, 1993)

\begin{abstract}
A multiple-scale asymptotic technique is used to study the propagation of slow hydromagnetic waves in a magnetostrophic regime for an incompressible, inviscid, perfectly conducting fluid of constant density in rotating cylindrical geometry. Such waves may play an important role in both the generation and behavior of the Earth's magnetic field. Knowledge of their behavior may be useful in studying the flows at the core-mantle boundary. The equations of motion are linearized about an ambient state of no motion with respect to rotating cylindrical coordinates and an azimuthal ambient magnetic field of the form $r^{n / 2}$ where $r$ is the radial distance in cylindrical coordinates. The waves may be trapped in a variety of ways while westward travelling modes may be unstable for $n>3$. These results extend the local results of ACHESON (1972) and are consistent with the numerical results of FEARN (1983).
\end{abstract}

\section{Introduction}

The purpose of this paper is to study the propagation of slow hydromagnetic waves in a magnetostrophic regime for an inviscid, incompressible perfectly conducting fluid of constant density in rotating cylindrical geometry, using a multiple-scale asymptotic technique. These waves may play an important role in the generation and behavior of the Earth's magnetic field. The work described here may be helpful in understanding the flows at the core-mantle boundary and can serve as a guide to numerical approaches to the problems of dynamics and stability of the Earth's outer core.

In a series of previous papers (LONDON, 1984, 1992a, 1992b) the author has studied slow hydromagnetic waves in spherical geometry assuming geostrophic balance in an attempt to model the propagation of such waves in the Earth's outer core. Since it is generally believed that the magnetic forces are at least as strong as the Coriolis force in the core, the author wished to extend his analyses to the magnetostrophic regime.

This work represents the author's initial attempt to extend the work of ACHESON (1972, $1973,1980,1983)$ to account for global variation of the ambient magnetic field as well as the presence of container boundaries. Acheson's work was local in nature and assumed a locally constant magnetic field, with no boundary effects, to derive conditions for magnetic field gradient instabilities. He found that instability can occur only if the waves are travelling westward and the ambient magnetic field grows locally faster than $r^{3 / 2}$ where $r$ is the distance from the axis of rotation. FEARN (1983) studied these results numerically and found a tendency for instabilities to concentrate approximately in the locally unstable region. Assuming general ambient magnetic fields proportional to $r^{n / 2}$, where $r$ is the radial distance in cylindrical coordinates, analytical results have been obtained here which are consistent with those of Acheson and Fearn. In addition, a rich variety of wave trapping occurs and an attempt is made to relate this trapping to the

*Partially supported by NSF grant EAR-9204460. 
behavior of the real geomagnetic field. In addition, these stability and wave trapping results are related in interesting ways.

The advantages of the asymptotic approach used here is in obtaining tractable, explicit quasi-global results for a very complicated problem. These result provide specific examples of phenomena which are suggested by the results of previous authors working on basically the same problem. They can act as an important guide for future numerical approaches to the problem of core waves and the geodynamo.

There are, however, certain fundamental limitations on the technique being used. It is restricted to the case of weak stability since it is necessary to assume that the time scale for the instability is an order of magnitude greater than the period of the waves. The geometry is obviously unrealistic for modelling the Earth's outer core, although the extension to a thin spherical shell should be relatively straightforward, very much in the manner of LONDON (1984). Magnetic diffusion is neglected so that the phenomena being analyzed must take place on a time scale less than the magnetic diffusion time scale of the ambient magnetic field.

In Section 2, we formulate the problem, describe the multiple scale asymptotic technique to be applied, discuss the assumptions which define the parameter regime and obtain a dispersion relation and conservation law for the slow hydromagnetic waves. These waves are analyzed in Section 3 for wave trapping and stability assuming ambient magnetic fields of the form $r^{n / 2}$. These results are discussed in Section 4.

\section{Assumptions, Scalings, and the Asymptotic Expansion}

Consider the motion of an incompressible, inviscid, perfectly conducting fluid of constant density with respect to a cylindrical coordinate system $(r, \theta, z)$ rotating with angular velocity $\Omega$.

At equilibrium, the fluid is at rest with respect to the rotating coordinate system in the presence of an ambient azimuthal magnetic field of the form

$$
\overrightarrow{b_{0}}=\left(0, b_{0}(r), 0\right) \text {. }
$$

The equations of motion and induction, linearized about this ambient state, may then be written

$$
\begin{gathered}
\vec{B}_{t}-\nabla \times\left(\vec{U} \times \vec{b}_{0}\right)=0, \\
\vec{U}_{t}+2 \Omega \vec{k} \times \vec{U}+(1 / \rho) \nabla P+\left(\vec{b}_{0} \times(\nabla \times \vec{B})+\vec{B} \times\left(\nabla \times \vec{b}_{0}\right)\right) /(\rho \mu)=0, \\
\nabla \cdot \vec{U}=0, \\
\nabla \cdot \vec{B}=0,
\end{gathered}
$$

where $\vec{U}$ is the velocity, $\vec{B}$ is the magnetic field, $P$ is the pressure, $t$ is the time, $\rho$ is the density and $\mu$ the magnetic permeability.

The assumption that the fluid is rapidly rotating (slow waves) and in magnetostrophic balance (Coriolis, magnetic and pressure forces of the same order of magnitude) requires that

$$
\Omega \gg 1 / T, B^{2} /(\rho \mu V R) \gg 1 / T, \psi /(\rho V R) \gg 1 / T,
$$

where $T$ is a characteristic period for the basic wave, $B$ a characteristic magnitude for the ambient magnetic field, $V$ a characteristic speed, $R$ a characteristic length, and $\psi$ a characteristic pressure. Under these assumptions, the inertial terms in (3) may be dropped so that the governing equations become

$$
\begin{gathered}
\vec{B}_{t}-\nabla \times\left(\vec{U} \times \vec{b}_{0}\right)=0, \\
2 \Omega \vec{k} \times \vec{U}+(1 / \rho) \nabla P+\left(\vec{b}_{0} \times(\nabla \times \vec{B})+\vec{B} \times\left(\nabla \times \vec{b}_{0}\right)\right) /(\rho \mu)=0,
\end{gathered}
$$




$$
\begin{aligned}
& \nabla \cdot \vec{U}=0, \\
& \nabla \cdot \vec{B}=0 .
\end{aligned}
$$

We now seek wave-like solutions to (7)-(10) of the form

$$
\begin{gathered}
\vec{U}=\left(u_{1}(r), u_{2}(r), u_{3}(r)\right) e^{i\left(-\omega^{*} t+k_{2} \theta+k_{3} z\right)}, \\
\vec{B}=\left(b_{1}(r), b_{2}(r), b_{3}(r)\right) e^{i\left(-\omega^{*} t+k_{2} \theta+k_{3} z\right)}, \\
P=p(r) e^{i\left(-\omega^{*} t+k_{2} \theta+k_{3} z\right)} .
\end{gathered}
$$

Note that it follows from (7) and (11) that (10) is satisfied as long as $\omega^{*} \neq 0$. After substituting (11) into (7)-(9), the remaining six dependent variables may be solved for in terms of the radial velocity $u_{1}(r)$. The following ordinary differential equation for $u_{1}(r)$ may then be derived:

$$
C_{2}(r) u_{1}^{\prime \prime}(r)+C_{1}(r) u_{1}^{\prime}(r)+C_{0}(r) u_{1}(r)=0,
$$

where

$$
\begin{aligned}
& C_{0}=\left(-4 \Omega^{2} \mu^{2} \omega^{* 2} r^{6} \rho^{2} k_{2}{ }^{2} k_{3}{ }^{2}-4 \Omega^{2} \mu^{2} \omega^{* 2} r^{8} \rho^{2} k_{3}{ }^{4}-12 \Omega \mu \omega^{*} r^{4} \rho k_{2}{ }^{3} k_{3}{ }^{2} b_{0}(r)^{2}\right. \\
& -8 \Omega \mu \omega^{*} r^{6} \rho k_{2} k_{3}{ }^{4} b_{0}(r)^{2}-k_{2}{ }^{6} b_{0}(r)^{4}+k_{2}{ }^{8} b_{0}(r)^{4}-5 r^{2} k_{2}{ }^{4} k_{3}{ }^{2} b_{0}(r)^{4} \\
& +2 r^{2} k_{2}{ }^{6} k_{3}{ }^{2} b_{0}(r)^{4}-2 r^{4} k_{2}{ }^{2} k_{3}{ }^{4} b_{0}(r)^{4}+r^{4} k_{2}{ }^{4} k_{3}{ }^{4} b_{0}(r)^{4}-2 r^{3} k_{2}{ }^{4} k_{3}{ }^{2} b_{0}(r)^{3} b_{0}^{\prime}(r) \\
& \left.-2 r^{5} k_{2}{ }^{2} k_{3}{ }^{4} b_{0}(r)^{3} b_{0}^{\prime}(r)\right) /\left(\mu \omega^{*} r^{2} \rho k_{2}{ }^{2}\left(k_{2}{ }^{2}+r^{2} k_{3}{ }^{2}\right)^{2} b_{0}(r)^{2}\right) \text {, } \\
& C_{1}=\frac{-k_{2}^{2} b_{0}(r)\left(k_{2}{ }^{2} b_{0}(r)-r^{2}{k_{3}}^{2} b_{0}(r)+2 r k_{2}{ }^{2} b_{0}^{\prime}(r)+2 r^{3} k_{3}{ }^{2} b_{0}^{\prime}(r)\right)}{\mu \omega^{*} r \rho\left(k_{2}{ }^{2}+r^{2}{k_{3}}^{2}\right)^{2}}, \\
& C_{2}=\frac{-k_{2}^{2} b_{0}(r)^{2}}{\mu \omega^{*} \rho\left(k_{2}^{2}+r^{2} k_{3}^{2}\right)} \text {. }
\end{aligned}
$$

We now assume

- that the vertical wavelength is much shorter than the azimuthal wavelength by requiring that

$$
k_{2}=m, k_{3}=\epsilon^{-1} l, 0<\epsilon \ll 1,
$$

- weak instability by admitting a small imaginary part for the frequency:

$$
\omega^{*}=\omega+i \epsilon \sigma
$$

and

- the WKB form for the solution:

$$
u_{1}(r)=e^{i \epsilon^{-1} S(r)} \sum_{n=0}^{\infty} A_{n}(r) \epsilon^{n},
$$

where $S(r)$ is a real-valued function. Equation (16) implies that the waves are short in the $r$ direction and that the wave number in the $r$ direction,

$$
k(r)=\frac{d S(r)}{d r}
$$

varies slowly with $r$. 
Perhaps the easiest way to understand (16) is to expand $S(r)$ about some point $r=r_{0}$ in the fluid domain. In a neighborhood of $r_{0}, \epsilon^{-1} S(r)=\epsilon^{-1} S\left(r_{0}\right)+\epsilon^{-1} S^{\prime}\left(r_{0}\right)\left(r-r_{0}\right)+\cdots$. It then follows from (16) that in a neighborhood of $r_{0}$, the local radial wave number is $\epsilon^{-1} k\left(r_{0}\right)=\epsilon^{-1} S^{\prime}\left(r_{0}\right)$ so that the local wave length is of the order $\epsilon c$, where $c$ is the radius of the outer core.

Before proceeding further, additional discussion of the assumptions (14)-(16) is in order. By assuming (14), we have restricted our analysis to a subspace of wave number space. Consequently, any solutions we find do not necessarily correspond to the most unstable modes, nor can stability be guaranteed for any region.

Equation (15) implies that while the time scale of the instability must be longer than that of the oscillations, it must still be shorter than the magnetic diffusion time scale since we have ignored magnetic diffusion in the governing equations (2)-(5). The assumption of weak instability (15) is made essentially for mathematical convenience: strong instability would require a complex phase function $S(r)$ which in turn would imply that the waves would be exponentially small or exponentially large.

That (15) is not an entirely unreasonable assumption may be seen from the Elsasser number $E l$ for the Core which measures the ratio of the diffusive timescale $\tau_{\eta}$ to the time scale for the slow waves studied in this paper $\tau_{s}$ (FEARN, 1988b). While GubBins and RoberTs (1987) note that the value of $E l$ is very uncertain, they estimate

$$
\frac{\tau_{\eta}}{\tau_{s}}=E l=20 .
$$

Therefore, assuming a time scale for the instabilities, $\tau_{i}$, which is significantly longer than $\tau_{s}$ but significantly shorter than $\tau_{\eta}$ is not unreasonable.

The assumptions (14) and (16) imply that radial and vertical wave lengths for the basic wave are much shorter than azimuthal wave length which we take to be on the order of the radius of the outer core (=3485 kilometers). We may estimate the short wave length scale by taking $\epsilon=\tau_{s} / \tau_{i}=1 / 5$ which is roughly the geometric mean of $\tau_{\eta}$ and $\tau_{s}$. Then the wave length of the basic wave is on the order of $1 / 5$ of the radius of the outer core or approximately 700 kilometers.

Substituting (14)-(16) into (12) and (13) gives a sequence of equations for $k(r)$ and the $A_{n}$ 's. The zeroth order equation may be solved for $k(r)$ to yield the dispersion relation:

$$
\begin{array}{r}
k(r)^{2}=l^{2}\left(4 \Omega^{2} \mu^{2} \omega^{2} r^{4} \rho^{2}+8 \Omega \mu \omega r^{2} \rho m b_{0}(r)^{2}+2 m^{2} b_{0}(r)^{4}\right. \\
\left.-m^{4} b_{0}(r)^{4}+2 r m^{2} b_{0}(r)^{3} b_{0}^{\prime}(r)\right) /\left(m^{4} b_{0}(r)^{4}\right)
\end{array}
$$

The first order equations may then be solved to obtain an ordinary differential equation for $A_{0}(r)^{2}$ which, upon solving, yields

$$
A_{0}(r)^{2}=\frac{C^{2} r}{|k(r)| b_{0}(r)^{2} m^{4} l^{2}} e^{-\sigma \int\left\{8 \Omega \mu r^{2} \rho\left(\Omega \mu \omega r^{2} \rho+b_{0}(r)^{2} m\right) l^{2} / b_{0}(r)^{4} m^{4} k(r)\right\} d r},
$$

where $C$ is a constant.

The general form for the leading order solution may be obtained as follows: (19) yields two solutions for $k(r)$, one associated with the positive square root of $k^{2}$, call it $k^{+}=k(r)$ and one associated with the negative square root, $k^{-}=-k(r)$. There are two solutions to Eq. (20), one associated with $k^{+}$, which we call $A_{0}^{+}(r)$, and one associated with $k^{-}$, called $A_{0}^{-}(r)$. It then follows from (11), (14), (15), (16) and (20) that to leading order, the radial velocity may be written

$$
u_{1}=\frac{e^{i \omega t+\epsilon \sigma t+i m \theta+i \epsilon^{-1} l z}}{m^{2}\left|b_{0}(r) l\right|} \sqrt{\frac{r}{|k(r)|}}
$$




$$
\begin{aligned}
& \times\left(C_{1} e^{i \epsilon^{-1} \int_{c}^{r} k(r) d r-\sigma \int_{c}^{r} \frac{4 \Omega \mu r^{2} \rho\left(\Omega \mu \omega r^{2} \rho+b_{0}(r)^{2} m\right) l^{2}}{b_{0}(r)^{4} m^{4} k(r)} d r}\right. \\
& \left.+C_{2} e^{-i \epsilon^{-1} \int_{c}^{r} k(r) d r+\sigma \int_{c}^{r} \frac{4 \Omega \mu r^{2} \rho\left(\Omega \mu \omega r^{2} \rho+b_{0}(r)^{2} m\right) l^{2}}{b_{0}(r)^{4} m^{4} k(r)} d r}\right)+O(\epsilon),
\end{aligned}
$$

where $C_{1}$ and $C_{2}$ are constants.

Equations (19), (20) and (21) are analyzed in the following section. In particular, we will

- analyze the spatial structure of the waves, particularly for the existence of singularities (caustics) and wave trapping,

- obtain an equation for the frequericies of oscillation, and

- study the stability of the waves.

\section{Wave Trapping, Singularities, and Stability}

We begin this section with a discussion of wave trapping. As will be seen below, wave trapping, singularities in the approximation, and the existence of unstable oscillations are all tied together.

It follows from (16) that waves propagate only in regions where $k(r)^{2}>0$ since $k(r)^{2}<0$ would imply that $k(r)=d S(r) / d r$ is imaginary so that the waves would be either exponentially small or unrealistically (exponentially) large.

We will analyze (19) for ambient magnetic fields of the form

$$
b_{0}(r)=r^{n / 2}, n \neq 2 .
$$

These fields will be of a form useful for comparison to the results of Acheson and Fearn. For $n=2$, $k(r)^{2}$ is a constant and no trapping occurs. This is the Malkus field; MALKUS (1967) recognized that essential simplifications occured in the problem for this choice of ambient magnetic field.

For ambient magnetic fields of the form $(22), k(r)^{2}$ in (19) may be written as a quadratic in terms of the variable $r^{n-2}$, and may have two positive zeros, $r_{1}$ and $r_{2}$, given by

$$
r_{1}=\left(\frac{-2 \Omega \mu \rho \omega}{m\left(2-\sqrt{2+m^{2}-n}\right)}\right)^{\frac{1}{n-2}}
$$

and

$$
r_{2}=\left(\frac{-2 \Omega \mu \rho \omega}{m\left(2+\sqrt{2+m^{2}-n}\right)}\right)^{\frac{1}{n-2}} .
$$

It follows from (19), (23) and (24) that wave trapping occurs for the ambient magnetic field $b_{0}=r^{n / 2}$ in a variety of ways which are summarized in Table 1 . (This list of wave regions is also a list of the regions in which the governing equations are hyperbolic.) In particular, it is interesting to note that wave trapping is more likely to occur for the westward drifting modes $c=\omega / m<0$ when the azimuthal wave number, $m$, is small.

It follows from (21) that the solution becomes singular at a caustic where the radial wave number, $k(r)$, vanishes. Generally speaking, such singularities are removable (see LoNDON and SHEN, 1979) but represent points in the fluid domain where the solution is amplified. The westward bias for the trapping of waves with small azimuthal wave number $m$ then suggests a possible mechanism for the westward drift. This possibility is discussed further in the following section.

To complete the analysis of the first order problem, we consider the application of lateral boundary conditions in order to determine the complex frequency $\omega^{*}=\omega+i \epsilon \sigma$. We begin by 


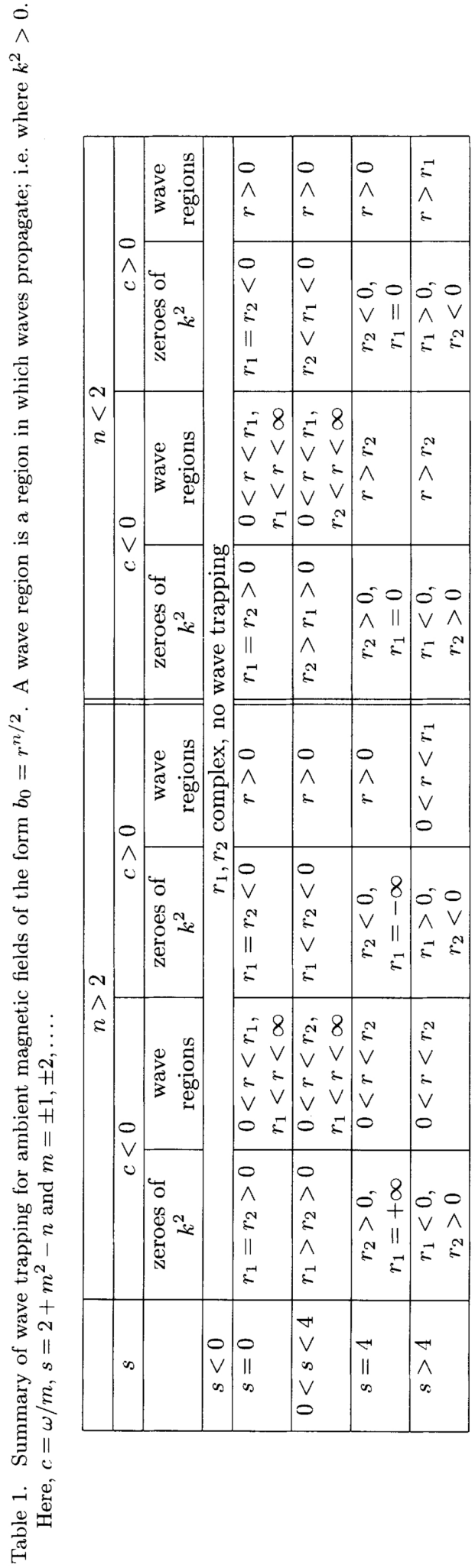


assuming that the wave region covers the entire fluid domain $r>0$ (i.e. no trapping) so that the lateral boundaries are cylinders at $r=c$ and $r=d$ where $0<c<d$. We will then generalize these results to the case of trapped waves.

In the case of no wave trapping, application of the lateral boundary conditions require that $u_{1}$ vanish at $r=c, d$. Applying these boundary conditions to $u_{1}$ in (21) requires that

$$
\begin{gathered}
i \epsilon^{-1} \int_{c}^{d} k(r) d r-\sigma \int_{c}^{d} \frac{4 \Omega \mu r^{2} \rho\left(\Omega \mu \omega r^{2} \rho+b_{0}(r)^{2} m\right) l^{2}}{b_{0}(r)^{4} m^{4} k(r)} d r=n \pi i \\
n=0, \pm 1, \pm 2, \ldots
\end{gathered}
$$

Equating the imaginary parts of (25) gives an equation for the resonance frequencies $\omega=$ $\operatorname{Re}\left(\omega^{*}\right)$.

Equating the real parts of (25) yields an equation which may also be obtained by constructing energy integrals (see FrIEDLANDER, 1987). For ambient magnetic fields of the form $b_{0}(r)=r^{n / 2}$, this equation becomes

$$
\sigma \int_{c}^{d} \frac{4 \Omega \mu \rho r^{2-2 n}\left(\Omega \omega \mu \rho r^{2}+r^{n} m\right) k_{3}^{2}}{m^{4} k(r)} d r=0 .
$$

Equation (26) can only be satisfied if either $\sigma$ vanishes, in which case the waves are stable, or if the integral vanishes, which would allow instability. But the integral can vanish only if the integrand changes sign in the wave region, i.e. if the numerator of the integrand has a zero in the wave region since, by definition, $k$ does not change sign in a wave region. This can only happen when $c=\omega / m<0$; i.e. for westward drifting modes.

The zero in the integrand of Eq. (26) may be written

$$
r_{0}=\left(\frac{-\Omega \mu \rho \omega}{m}\right)^{\frac{1}{n-2}} \text {. }
$$

Recall that $m= \pm 1, \pm 2, \ldots$, so that $s \geq 0$ for $n \leq 3$. It then follows from (23), (24), (27) and Table 1 that $r_{0}$ can never be in a wave region, and instability is therefore impossible, if $n \leq 3$. These are essentially ACHESON's (1972) results extended to variable (non-local) ambient magnetic fields, and they are basically consistent with the numerical results of FEARN (1983).

We now wish to extend (25) to the cases of wave trapping. In these cases, caustics appear and may act as boundaries of the wave regions, depending on the values of $c$ and $d$. Techniques for dealing with asymptotic eigenvalue problems involving caustics in the fluid domain were developed by KELLER and RUBINOW (1960), and exploited by many authors. A useful reference in this regard is the paper by SHEN et al. (1968) relating to the spectra of water waves.

We assume that in the case where there is more than one wave region in the fluid, as can happen in this problem (see Table 1), the two regions are independent (i.e. no energy is exchanged between the regions) and the spectral calculations are independent for the two regions. Then the spectral/stability formula, (25), modified to account for the presence of caustics as boundaries, may be written

$$
\begin{gathered}
i \epsilon^{-1} \int_{c}^{d} k(r) d r-\sigma \int_{c}^{d} \frac{4 \Omega \mu r^{2} \rho\left(\Omega \mu \omega r^{2} \rho+b_{0}(r)^{2} m\right) l^{2}}{b_{0}(r)^{4} m^{4} k(r)} d r=\left(n+\frac{1}{4} m_{l}\right) \pi i, \\
n=0, \pm 1, \pm 2, \ldots,
\end{gathered}
$$

where $m_{l}=0,1$, or 2 is the number of caustics acting as wave region boundaries; $r=c$ and $r=d$ could be caustics, depending on the geometry of the problem. The stability is still determined 
from (26), and again the zero of the numerator of the integrand, $r_{0}$, can never be in a wave region when $n \leq 3$. Therefore, the basic results remain the same: instability can occur only if $b_{0}(r)$ increases with $r$ faster than $r^{3 / 2}$ and only for westward drifting modes.

Finally, FEARN's (1983) observation that instabilities tend to concentrate approximately in locally unstable regions is supported by the following argument: Suppose $d b_{0}(r)^{2} / d r$ is both positive and sufficiently large in some region, and that $\omega / m<0$. It then follows from (19) and (25) that this region is included in a wave region and that the integrand in (25) may change sign in that region, thus admitting the possibility of unstable waves there.

\section{Discussion}

While we have seen that the asymptotic approach used to obtain the above results has certain drawbacks, we have also noted that the assumptions underlying this approach may not be entirely unreasonable. It is therefore of some interest to see if there is any indication that the westward drift has a structure consistent with the waves found here: a long length scale in the azimuthal direction (on the order of thousands of miles) and a short length scale in the longitudinal direction (on the order of hundreds of miles). MERRILL and MCELHINNy (1983) compared the vertical component of the non-dipole field for 1829 and 1980. The central African anomaly, which was clearly drifting westward, appears to be associated with a structure somewhat like that which we are seeking. On the other hand, there are locations where the structure is inverted (long length scale in the azimuthal direction and short in the longitudinal) but the motion seems less obvious.

As noted in the previous section, there is a bias toward westward propagation for stable waves with small azimuthal wave number. If stable waves of the sort discussed in this paper were responsible for the westward drift, we might expect the data to show banded or layered movement patterns. The charts in Merrill and McElhinny referred to above appear to show a more pronounced drift in a band near the equator. On the other hand, Merrill and McElhinny display data from YUKUTAKE and TACHINAKA (1969) who divide the non-dipole field into its drifting and non-drifting components; the drifting components show no apparent banding.

The discussion of the preceding two paragraphs is meant only as a plausibility argument for one possible westward drift mechanism out of many. Such mechanisms include wave propagation, bulk motion of the fluid, retrograde motion of the core with respect to the mantle due to electromagnetic coupling, etc. All of these mechnisms may be operative to some degree.

Two significant drawbacks to our approach have been the assumptions of weak instability and cylindrical geometry. We anticipate that it is possible to overcome both of these restrictions. We believe the restriction to weak instability could be overcome by attacking the problem in a manner similar to that of LOZANO and MEYER (1976). As to extending these results to spherical geometry, the most fruitful approach would probably be to begin by applying the method of Section 2 to a thin spherical shell in order to model some of the effects of the spherical geometry.

Because of the important role played by resistive instabilities (FEARN, 1984, 1985, 1988; FEARN and WeIGLHOFER, 1991) and viscous boundary layers (GILMAN and BENTON, 1968; Benton and Loper, 1969; Loper and Benton, 1970; Gilman, 1971; Loper, 1971; Benton, 1973), we would eventually like to extend these results to include the effects of ohmic and viscous diffusion. SHEN (1976) has applied an asymptotic technique of the type used here to the problem of surface waves in a viscous fluid. Some variant of his approach may be applicable to the present problem. Extension of this work to model the real core-mantle boundary will require that both spherical and diffusive effects be incorporated into a boundary layer analysis where the magnetostrophic approximation may no longer be valid and the boundary conditions will be different.

Finally, the effects of buoyancy (as a possible energy source for these waves) and differential rotation (FEARN and Proctor, 1983a, b) eventually need to be accounted for. 


\section{REFERENCES}

ACHESOn, D. J., On the hydromagnetic stability of a rotating annulus, J. Fluid Mech., 52, 529-541, 1972.

ACHESON, D. J., Hydromagnetic wavelike instabilities in a rapidly rotating stratified fluid, J. Fluid Mech., 61, $609-624,1973$.

ACHESON, D. J., Stable density stratification as a catalyst for instability, J. Fluid Mech., 96, 723-733, 1980.

ACHESON, D. J., Local analysis of thermal and magnetic instabilities in a rapidly rotating fluid, Geophys. Astrophys. Fluid Dyn., 27, 123-136, 1983.

Benton, E. R., Nonlinear hydrodynamic and hydromagnetic spin-up driven by Ekman-Hartmann boundary layers, J. Fluid Mech., 57, 337-360, 1973.

Benton, E. R. and D. E. Loper, On the spin-up of an electrically conducting fluid. Part 1. The unsteady hydromagnetic Ekman-Hartmann boundary layer problem, J. Fluid Mech., 39, 561-586, 1969.

FEARN, D. R., Hydromagnetic waves in a differntially rotating annulus I. A test of local stability analysis, Geophys. Astrophys. Fluid Dyn., 27, 137-162, 1983.

FEARN, D. R., Hydromagnetic waves in a differentially rotating annulus II. Resistive instabilities, Geophys. Astrophys. Fluid Dyn., 30, 227-239, 1984.

FEARN, D. R., Hydromagnetic waves in a differentially rotating annulus III. The effect of an axial field, Geophys. Astrophys. Fluid Dyn., 33, 185-197, 1985.

FEARN, D. R., Hydromagnetic waves in a differentially rotating annulus IV. Insulating boundaries, Geophys. Astrophys. Fluid Dyn., 44, 55-75, 1988a.

FEARn, D. R., Instabilities of toroidal magnetic fields, in Structure and Dynamics of the Earth's Deep Interior, edited by D. E. Smylie and R. Hide, pp. 129-133, Geophysical Monograph 46, IUGG Volume I, 1988 b.

FEARn, D. R. and M. R. E. Proctor, Hydromagnetic waves in a differentially rotating sphere, J. Fluid Mech., 128, 1-20, 1983a.

FEARN, D. R. and M. R. E. Proctor, The stabilizing role of differential rotation on hydromagnetic waves, $J$. Fluid Mech., 128, 21-36, 1983b.

FEARN, D. R. and W. S. WeIGlhofer, Magnetic instabilities in rapidly rotating spherical geometries I. From cylinders to spheres, Geophys. Astrophys. Fluid Dyn., 56, 159-181, 1991.

Friedlander, S., Hydromagnetic waves in the Earth's fluid core, Geophys. Astrophys. Fluid Dyn., 39, 315-333, 1987.

Gilman, P. A., Instabilities of the Ekman-Hartmann boundary layer, Phys. Fluids, 14, 7-12, 1971.

Gilman, P. A. and E. R. BEnton, Influence of an axial magnetic field on the steady linear Ekman boundary layer, Phys. Fluids, 11, 2397-2401, 1968.

Gubbins, D. and P. H. Roberts, Magnetohydrodynamics of the Earth's core, in Geomagnetism, Vol. 2, edited by J. A. Jacobs, 306 pp., Academic Press, London, 1987.

Keller, J. B. and S. I. Rubinow, Asymptotic solutions of eigenvalue problems, Ann. Phys., 9, 24-75, 1960.

LONDon, S. D., Slow hydromagnetic oscillations in a rotating spheroidal shell, Geophys. Astrophys. Fluid Dyn., 28, 205-222, 1984.

London, S. D., Hydromagnetic waves in a rotating sphere, Geophys. Astrophys. Fluid Dyn., 63, 91-109, 1992a.

LONDON, S. D., A uniform asymptotic approximation for unstable hydromagnetic waves in a rotating sphere, Geophys. Astrophys. Fluid Dyn., 67, 185-198, 1992b.

London, S. D. and M. C. Shen, Free oscillation in a rotating spherical shell, Phys. Fluids, 22, 2071-2080, 1979.

LOPER, D. E., Hydromagnetic spin-up of a fluid confined by two flat electrically conducting boundaries, J. Fluid Mech., 50, 609-623, 1971.

Loper, D. E. and E. R. Benton, On the spin-up of an electrically conducting fluid. Part 2. Hydromagnetic spin-up between infinite flat insulating plates, J. Fluid Mech., 43, 785-799, 1970.

Lozano, C. and R. E. Meyer, Leakage and response of waves trapped by round islands, Phys. Fluids, 19, 1075$1088,1976$.

Malkus, W. V. R., Hydromagnetic planetary waves, J. Fluid Mech., 28, 793-802, 1967.

Merrill, R. T. and M. McElhinny, The Earth's Magnetic Field, Academic Press, London, 1983.

Shen, M. C., Surface waves on viscous fluid of variable depth, Phys. Fluids, 19, 1669-1675, 1976.

Shen, M. C., R. E. MEYeR, and J. B. Keller, Spectra of water waves in channels and around islands, Phys. Fluids, 11, 2289-2304, 1968.

Yukutake, T. and H. TAChinaka, Separation of the earth's magnetic field into drifting and standing parts, Bull. Earthquake Res. Inst., 47, 65-97, 1969. 